\title{
Molecular design of a new class of inhibitors for ion channel of influenza protein
}

\author{
Y.N. Vorobjev, O.S. Fedorova \\ Institute of Chemical Biology and Fundamental Medicine SB RAS, Novosibirsk, Russia \\ Novosibirsk State University, Novosibirsk, Russia \\ *e-mail:ynvorob@niboch.nsc.ru
}

Key words: M2 protein, ionization, drug binding, histidine ionization

Motivation: A design of novel anti-influenza drugs is a task of great importance due to a capability of influenza viruses to infect fast a large human population by occasional cross of inter-species barriers and to rapid mutate.

Methods: Transport of $\mathrm{H}^{+}$ion through ion channel of protein $\mathrm{M} 2$ of cell membrane can be blocked by drug molecule bound inside of ion channel. A new class of molecular blockers is suggested. Binding mode and binding energies are calculated for a set of novel molecular structures constructed on the base of diazobicyclooctane.

Results: A set of molecular structures as a derivatives of leading compound is suggested. Binding modes and energies of binding are calculated by method of hierarchical blind docking [1]. A molecular structure of an optimal molecular blocker is suggested.

Acknowledgements: The work is supported by grant RFFI No. 18-04-00005 and by Russian State funded project program (No. VI.57.1.2, 0309-2018-0001).

References

1. Vorobjev Y.N. (2010) J Comput Chem. 31:1080-1092. 